\title{
CT for upper abdominal pathology - is imaging of the pelvis necessary?
}

\author{
C Ackermann, MB ChB, MMed Rad (D), MRCS (UK) \\ S Andronikou, MB BCh, FCRad (D), FRCR, PhD \\ A Erlank, $M B C h B$ \\ J J Parsons, MB ChB, MMed Rad (D) \\ G Cilliers, MB ChB, MMed Rad (D) \\ Department of Radiology, University of Stellenbosch and Tygerberg Hospital
}

\section{Abstract}

Background. Current practice at our institution for routine abdominal CT includes coverage from the diaphragm to the symphysis pubis and therefore includes pelvic organs. Limited upper abdominal imaging exists in other modalities, and tailoring the examination to pathology will result in higher positive yield.

Objective. To determine if the pelvic component of a routine abdominal CT scan contributes to the final diagnosis in organ-specific upperabdominal pathology.

Methods. This was a retrospective study spanning a 14-month consecutive period; all abdominal CTs for organ-specific upper-abdominal pathology were included. There were no age or gender limitations. Patients with multi-organ involvement such as lymphoma and TB were excluded. The consultant radiologists' reports were evaluated for the indication, preceding investigations, presence of pelvic pathology and final CT diagnosis.

Results. Of 133 CT studies done, 116 did not show any abnormality in the pelvis. In 3 cases there were pelvic abnormalities that contributed to making the final diagnosis. Independent review of these cases by 4 consultant radiologists with masking of the pelvic cuts did not influence the final outcome. In 5 cases free fluid was noted and in 9 cases there were incidental findings with no impact on the final diagnosis.

Conclusions. It is not essential to include the pelvis in the field of radiation in scanning specific upper-abdominal pathology, with the exclusion of staging a known renal mass and imaging renal calculi.

\section{Introduction}

Current practice at our institution for routine abdominal CT includes coverage from the diaphragm to the symphysis pubis and therefore includes pelvic organs.

This study aimed to determine whether the pelvic component of a routine abdominal CT for upper-abdominal pathology contributes to the final diagnosis, and if not to exclude it from the protocol and reduce ionizing radiation to the pelvis as well as improve patient throughput (both beneficial to the patient).

The most common indications for abdominal CT at our institution include: obstructive jaundice, pancreatitis, complicated renal cysts and liver lesions - all upper-abdominal pathology. The question arises whether the pelvis receives unnecessary radiation in many patients. ${ }^{1}$ Limited upper-abdominal imaging is common practice and is used in other modalities such as US and MRI. ${ }^{2,3}$ Many protocols for specific upper-abdominal pathology exist ${ }^{4}$ and wide variations of these are in use. ${ }^{5}$ Federle and Blachar ${ }^{6}$ believe that a standard abdominal CT does not exist and that all investigations should be tailored to the pathology. It has also been found that abdominal CT performed for generalised abdominal pain with poor clinical examination and laboratory results has a very low positive yield. ${ }^{7}$ It is mainly in oncology and staging examinations that the pelvis is included as a routine part of the study.

\section{Objective}

The aim of the study was to determine if the pelvic component of a routine abdominal CT contributes to the final diagnosis in organ-specific upper-abdominal pathology.

\section{Methods}

A retrospective study was done spanning a consecutive 14-month period; all abdominal CTs for organ-specific upper-abdominal pathology (hepatobilliary, pancreatic, splenic, adrenal and renal abnormalities) were included. The mean age was 51.6 years. The oldest patient was 91 and the youngest patient 2 years of age.

Abdominal CTs where multi-organ involvement was suspected, i.e. lymphoma, abdominal TB and trauma, were excluded.

The request forms and consultant radiologist's reports were evaluated for: (i) indications and presenting symptoms; (ii) preceding investigations, specifically abdominal US; (iii) findings of US investigation with specific reference to findings in the pelvis; (iv) the presence or absence of pelvic pathology on CT; and (v) the final CT diagnosis by the radiologist.

\section{Results}

One hundred and thirty-three CT studies met the inclusion criteria. Of these, 116 did not show any abnormality in the pelvis.

In 3 cases pelvic abnormalities contributed to making the final diagnosis.

The first patient had chronic pancreatitis and was scanned for evaluation of a possible pancreatic mass. Matted bowel loops were found in the pelvis. Complicated pancreatitis or possible underlying malignancy of the pancreas was suggested as a diagnosis.

The second patient had hepatocellular carcinoma. No pathology was noted in the pelvis, but the right iliac wing and L5 vertebral body demonstrated metastases.

The third patient had diabetes mellitus and a mass in the right kidney on US. Hydroureteronephrosis was present, with multiple renal abscesses. A level of obstruction was identified on CT due to an enlarged uterus compressing the right ureter.

To evaluate the ability to make the correct diagnosis in the above 3 cases without the pelvic images, 4 independent consultant radiologists reported the cases with masking of the pelvic cuts. The results are presented in Table I, and indicate that in all 3 cases the individual reports contained the same conclusion, and were able to confirm the diagnosis as reported on the initial study. 


\section{ORIGINAL ARTICLE}

\begin{tabular}{|c|c|c|c|c|}
\hline Patient & Radiologist 1 & Radiologist 2 & Radiologist 3 & Radiologist 4 \\
\hline \multirow[t]{3}{*}{1} & 1) Lymphoma & 1) Pancreatitis & 1) Exudative pancreatitis & 1) Pancreas head CA \\
\hline & 2) Pancreas head $C A$ & \multirow[t]{2}{*}{ 2) Pancreas head CA } & & 2) Pancreatitis \\
\hline & 3) Focal pancreatitis & & & \\
\hline \multirow[t]{2}{*}{2} & 1) Liver and bony metastases & 1) Hepatocellular CA & 1) Hepatocellular CA & \multirow[t]{2}{*}{ 1) Hepatocellular CA } \\
\hline & 2) Hepatocellular CA & 2) Liver metastases & 2) Liver metastases & \\
\hline 3 & $\begin{array}{l}\text { 1) Pyonephrosis, hydro- } \\
\text { ureteronephrosis }\end{array}$ & $\begin{array}{l}\text { 1) Pyonephrosis, hydro- } \\
\text { ureteronephrosis }\end{array}$ & $\begin{array}{l}\text { 1) Pyonephrosis, hydro- } \\
\text { ureteronephrosis }\end{array}$ & $\begin{array}{l}\text { 1) Renal abscess, hydro- } \\
\text { ureteronephrosis }\end{array}$ \\
\hline \multicolumn{5}{|c|}{$\begin{array}{l}\text { The differential diagnoses are presented in most likely order where applicable. } \\
\text { Final diagnosis: 1. Complicated pancreatitis. 2. Hepatocellular carcinoma. 3. Pyonephrosis, hydroureteronephrosis. }\end{array}$} \\
\hline
\end{tabular}

\begin{tabular}{|c|c|c|}
\hline \multicolumn{3}{|c|}{ Table II. Indications for CT scanning } \\
\hline Symptoms / organ related & Organ-specific indications & Number (\%) \\
\hline \multirow[t]{8}{*}{ Liver } & Hepatocellular carcinoma & $4(3)$ \\
\hline & Hepatoblastoma & $4(3)$ \\
\hline & Liver mass & $3(2.2)$ \\
\hline & Metastases & $2(1.5)$ \\
\hline & Liver abscess & $1(0.7)$ \\
\hline & Liver cirrhosis & $1(0.7)$ \\
\hline & Hepatospenomegaly & $3(2.2)$ \\
\hline & Portal hypertension & $1(0.7)$ \\
\hline Spleen & Splenomegaly & $1(0.7)$ \\
\hline \multirow[t]{2}{*}{ Pancreas } & Pancreatitis (acute/chronic) & $9(6.8)$ \\
\hline & Pancreas head carcinoma & $4(3)$ \\
\hline Adrenals & Adrenal mass & $2(1.5)$ \\
\hline \multirow[t]{5}{*}{ Kidneys } & Renal cell carcinoma & $25(18.7)$ \\
\hline & Cysts & $4(3)$ \\
\hline & Abscess/pyonephroses & $3(2.2)$ \\
\hline & Renal calculi & $2(1.5)$ \\
\hline & Angiomyolipoma & $1(0.7)$ \\
\hline \multirow[t]{2}{*}{ Stomach } & Stomach outlet obstruction & $2(1.5)$ \\
\hline & Stomach carcinoma & $2(1.5)$ \\
\hline \multirow[t]{3}{*}{ Gallbladder/bileducts } & Gallbladder carcinoma & $2(1.5)$ \\
\hline & Cholangiocarcinoma & $1(0.7)$ \\
\hline & Cholangitis & $1(0.7)$ \\
\hline Obstructive jaundice & & $25(18.7)$ \\
\hline Right hypochondrial pain & & $9(6.8)$ \\
\hline Left hypochondrial pain & & $1(0.7)$ \\
\hline Right flank mass & & $3(2.2)$ \\
\hline Right flank pain & & $4(3)$ \\
\hline Epigastric pain & & $5(3.7)$ \\
\hline Epigastric mass & & $7(5.2)$ \\
\hline Gastrointestinal haemorrhage & & $1(0.7)$ \\
\hline
\end{tabular}


In 5 cases (3.7\%), free fluid was noted in the pelvis which did not influence the final diagnosis, but contributed to a complete evaluation.

In 9 cases (6.8\%), incidental findings were noted on CT with no impact on the final diagnosis. These were ovarian cysts $(N=4)$, prostate calcifications $(N=2)$, uterine myomas $(N=2)$, and fluid in the uterus $(N=1)$.

Prior to CT investigation, 89 patients had US examinations (67\%); of these only 59 could be retrieved. In 56 cases no pelvic pathology was reported, 2 cases demonstrated enlarged prostates and in 1 case free fluid was noted in the pelvis.

The commonest requests for CT scanning were evaluation for obstructive jaundice $(N=25,18.8 \%)$ and complicated renal cysts/exclusion of renal cell carcinoma $(N=25,18.8 \%)$. Liver pathology as a group also ranked high on the request list (Table II).

The final diagnoses are listed in Table III, of which the most common were pancreas head carcinoma $(N=14,10.5 \%)$, pancreatitis $(N=$ $13,9.8 \%)$, renal cell carcinoma $(N=12,9 \%)$ and cholangiocarcinoma $(N=11,8.2 \%)$.

The indeterminate or non-specific findings comprised $33 \%$ of the group $(N=44)$, and there was a total of 12 normal studies $(9 \%)$.

\section{Discussion}

With the technical advances in CT scanning, the quality of imaging has improved dramatically, especially with regard to: Decrease in scanning time, elimination of respiratory misregistration artifacts, imaging during optimal parenchymal as well as vascular contrast enhancement, ${ }^{8}$ and reconstructing images retrospectively. ${ }^{5}$

It is also generally accepted that previous poor visualisation of the liver and pancreas can be ascribed to the use of a general standard abdominal CT protocol for upper-abdominal pathology. ${ }^{6}$

In this study most of the requests for abdominal CT were aimed at resolving specific problems. In these cases the patients had several investigations prior to scanning to identify the pathology more accurately and to limit the list of differential diagnoses.

This supports the findings of previous studies looking at the positive yield of abdominal CT scans. ${ }^{79}$ In the light of this it can be concluded that when faced with a specific request / problem, the radiologist's approach must be tailored to address this and plan the CT scan accordingly. ${ }^{5}$ Recently, it was noted that up to $40 \%$ of all CT examinations in children have questionable indications. Clinical information will help guide the radiologists' choices of imaging modalities (e.g. sonography or MR imaging replacing CT) and protocols or techniques. ${ }^{10}$

For this reason, we wanted to evaluate preceding US examinations and establish the correlation of pathology noted in the pelvis on US with abnormalities on CT, and whether US can be used as a good sorting tool. ${ }^{11,1}$

Eighty-nine patients had US prior to scanning. In the 56 retrieved studies no pathology was demonstrated in the pelvis, which correlated well with the CT scan. An incidental finding of an enlarged prostate in 1 case was not reported on the CT scan.

In 5 cases pelvic pathology noted on CT was not reported on the US, namely incidental prostate calcifications $(N=2)$, ovarian cyst ( $N$ $=1)$, and free fluid $(N=2)$. It is accepted that US is both sensitive and specific for detecting free fluid in the abdomen and pelvis. ${ }^{13,14}$ Free fluid was missed in the pelvis of 2 patients. Possible reasons for the discrepancy could be a time difference between US and CT scan, operator dependence and poor evaluation of the pelvis as directed by a request for upper-abdominal pathology.

The most common requests for CT scan at our institution were evaluation of obstructive jaundice and complicated renal cysts.

The most common diagnoses made were adenocarcinoma of the pancreas, pancreatitis, renal cell carcinoma and cholangiocarcinoma. The literature quoted deals mainly with common imaging findings in specific upper-abdominal pathology and some advice on scanning protocols. One article ${ }^{10}$ pertinently discusses the issue of inclusion of the pelvis in the field of imaging, mainly relating to paediatric patients. Frush $^{10}$ states that the CT scan can be designed to answer a specific question and this means that pelvic scanning is not always necessary with an abdominal scan, and that follow-up CT examinations do not necessarily need to be full abdomen or chest examinations. He also makes the point that CT likely accounts for the single largest radiation exposure after background. This data precedes the newest MDCT, and it is likely that the increasing use will continue to accelerate. ${ }^{10}$

Review of the literature states that the most common pancreatic

\begin{tabular}{|lll|}
\hline \multicolumn{2}{|c|}{ Table III. Most common diagnoses made in this study } & \% \\
\hline Diagnosis & N & 10.5 \\
\hline Pancreas head carcinoma & 14 & 9.8 \\
Pancreatitis & 13 & 9 \\
Renal cell carcinoma & 12 & 8.3 \\
Cholangiocarcinoma & 11 & 6.8 \\
Hepatocellular carcinoma & 9 & 6 \\
Simple renal cysts & 8 & 3.7 \\
Autosomal-dominant polycystic kidney disease and hepatoblastoma & 5 & 2.2 \\
Renal abscess, echinococcus cysts, liver metastases and stomach carcinoma & 3 & 1.5 \\
Liver abscess, adrenal adenoma and gallbladder carcinoma & 2 & 33 \\
Other & 44 & 9 \\
Normal & 12 & \\
\hline
\end{tabular}



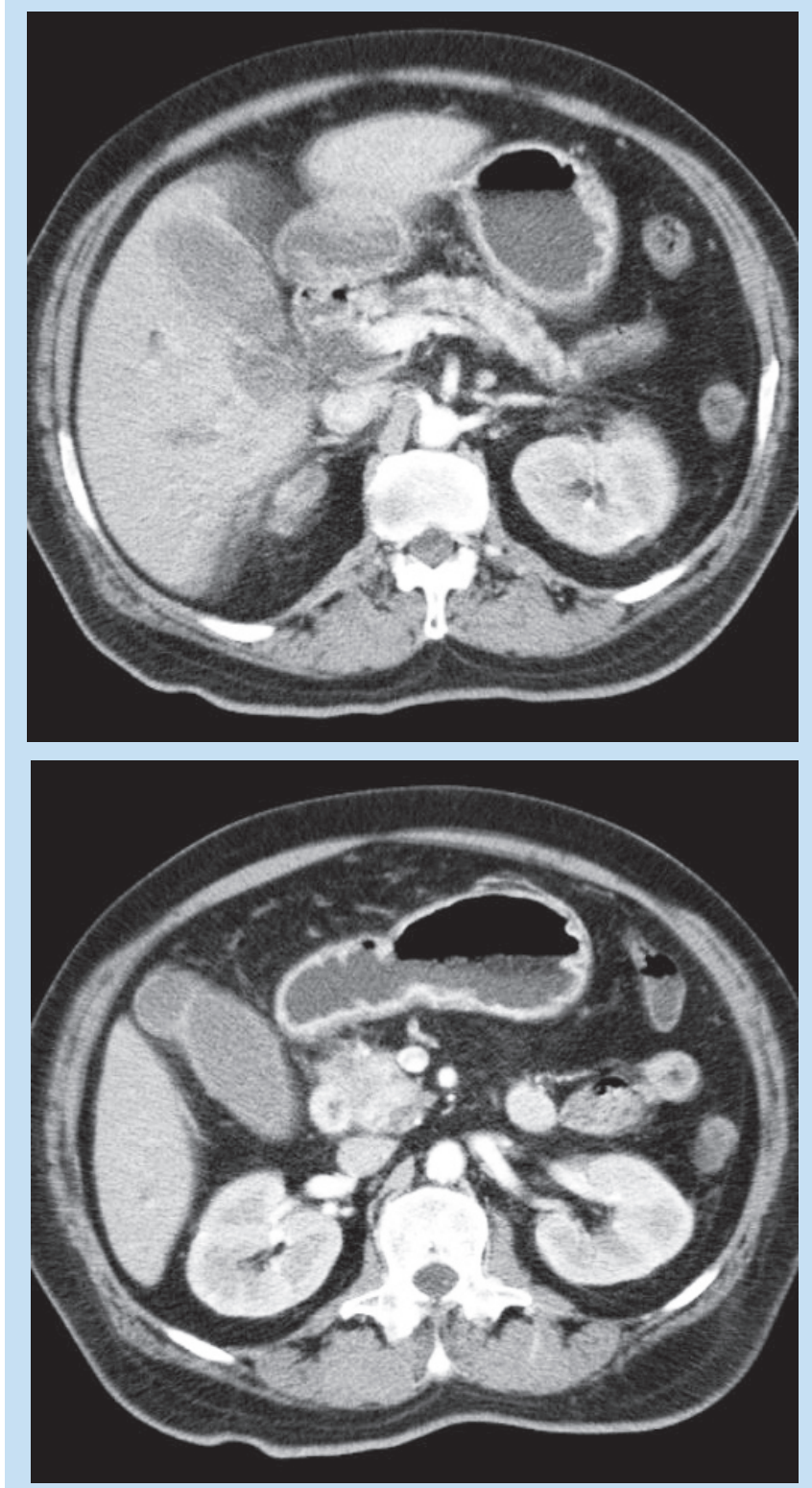

Figs $1 a$ and $b$. Adenocarcinoma of the uncinate/head of pancreas. Fig. 1a. Abdominal CT in the late arterial phase demonstrating a dilated pancreatic duct as well as common bile duct. The body and tail of the pancreas appear atrophic.

Fig. $1 \mathrm{~b}$. An ill-defined low-density mass is present in the head/uncinate process of the pancreas. Note clear separation from the SMA and SMV but close approximation to the duodenum. No regional lymphadenopathy is present.

pathology demonstrated on CT is pancreatitis and pancreatic adenocarcinoma. ${ }^{15}$ Adenocarcinoma is the most common malignant pancreatic tumour, affecting the head of the pancreas in $60-70 \%$ of cases. ${ }^{16}$ Today, $\mathrm{CT}$ is the most commonly used imaging method in the assessment of pancreatic tumours. ${ }^{17}$ On imaging the primary tumour is a hypoattenuating mass, usually well visualised on the pancreatic phase (a circulation phase corresponding in timing to the late arterial/portal vein inflow phase, when the pancreatic parenchyma is enhanced more than hepatic parenchyma $)^{15}$ (Figs. 1a and b). Cephalocaudate coverage for the pancreatic phase, as used by W Dennis Foley, is from the diaphragm to the

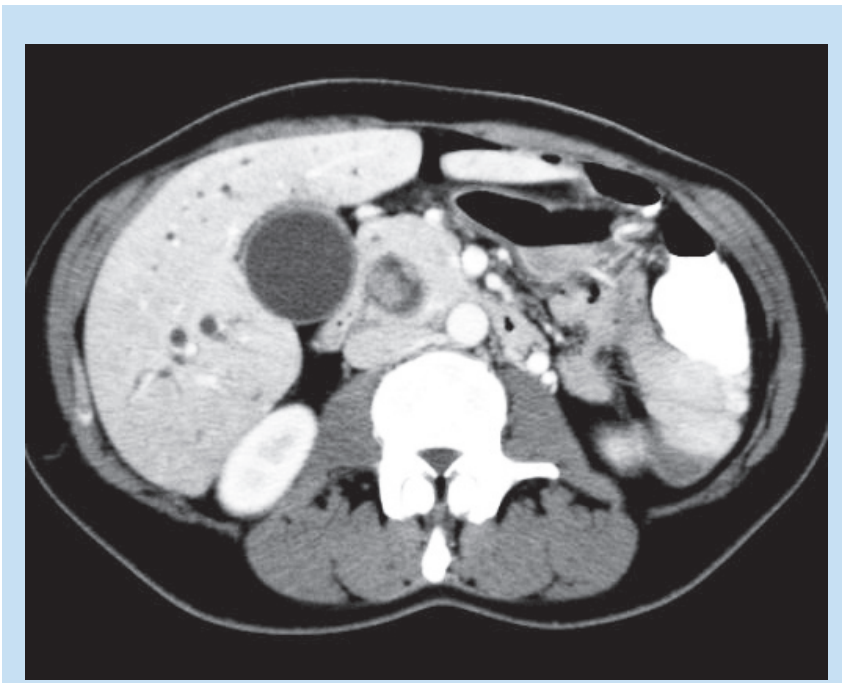

Fig. 2. Abdominal CT in the portovenous phase demonstrating a distended gallbladder, severe intra/extra hepatic bile duct dilatation and a lobulated, enhancing mass in the distal common bile duct in keeping with a cholangiocarcinoma.

inferior margin of the pancreas, and is usually less in extent than for a hepatic MDCT study.

Imaging is used to assess resectability, extrapancreatic spread and metastases. Nonresectable tumours invade the peripancreatic tissue planes to encase the celiac axis and superior mesenteric artery, the superior mesenteric vein and portal vein confluence, as well as the hepatoduodenal ligament and hepatic hilum. Other signs of nonresectability include peripancreatic lymphadenopathy, hepatic metastases, and peritoneal carcinomatosis, the latter usually associated with either infiltration of the root of the mesentery or the transverse mesocolon. ${ }^{15}$ None of the above important findings relate to possible abnormalities in the pelvis.

We confirmed this in our study, with no significant pathology noted in the pelvis of all the patients finally diagnosed with adenocarcinoma of the pancreas. In one patient with pelvic abnormality on CT (matted bowel loops), free fluid was documented on the preceding US and could have alerted the radiologist to pathology in the pelvis and subsequent alteration of scanning method if deemed necessary. Masking of the pelvic cuts did not alter the outcome of the final CT report.

In acute pancreatitis, the major imaging findings are pancreatic necrosis, peripancreatic effusion and pseudocyst, and the possible complications of peripancreatic venous thrombosis and gastrointestinal fistulisation. A double pass pancreatic technique is advised consisting of a pancreatic and hepatic phase, with coverage as described above for adenocarcinoma of the pancreas. ${ }^{15}$

Malignant biliary obstruction is usually secondary to pancreatic adenocarcinoma or is due to cholangiocarcinoma of the common bile, or common hepatic duct (including the bifurcation, collectively called hilar), or the intrahepatic bile ducts (peripheral). Cholangiocarcinoma is an adenocarcinoma that arises from the bile duct epithelium and is the second most common primary hepatobiliary cancer, after hepatocellular cancer, accounting for $5-30 \%$ of all primary hepatic malignant tumours. ${ }^{18,19}$ Frequently these patients will have had sonography, ERCP or MRCP, with the level of obstruction having been identified and the 
likely diagnosis of pancreatic adenocarcinoma or cholangiocarcinoma established. ${ }^{15}$ Cholangiocarcinomas may be limited to the bile duct and adjacent periductal tissues, being either infiltrating or polypoid in configuration (Fig. 2). Alternatively, the tumour may present as a mass lesion at the hepatic hilum and be associated with perihepatic adenopathy. ${ }^{15}$ The major issue of imaging this tumour is to determine whether the tumour is resectable, ${ }^{20}$ and with local infiltration they have a dismal prognosis if left untreated, with a mean survival of approximately 3 months after the initial presentation. ${ }^{21}$ Information needed is therefore concentrated on the liver, with imaging of the pelvis becoming superfluous. WD Foley believes the average cephalocaudate distance of the liver, approximately $16 \mathrm{~cm}$, allows the cephalocaudate hepatic span, in the majority of patients to be accomplished in $20 \mathrm{~cm}$ of scan coverage. In addition the hepatic phase is extended to include the whole abdomen, and possibly pelvis, to evaluate for extrahepatic disease. ${ }^{15}$ In one of our patients diagnosed with hepatocellular carcinoma pelvic bone and lumbar vertebral metastases (L5), would not have been diagnosed had the pelvis not been included. These findings were missed by 3 of the observing radiologists when reporting the masked CT scans, but one radiologist observed the L5 metastases. Known CT protocols for the evaluation of possible liver mass/ hepatocellular carcinoma do not include the pelvis routinely. ${ }^{22}$

Renal cell carcinoma (RCC) is the most common cancer of the kidney, accounting for approximately $2-3 \%$ of adult malignancies. ${ }^{23}$

Radical cancer surgery remains the only curative treatment in localised and advanced RCC. Therefore, preoperative imaging is most important for planning of the surgical approach and strategy. The aim of preoperative imaging in RCC is to differentiate benign from malignant lesions, to adequately assess tumour size, localisation and organ confinement, to identify lymph node and/or visceral metastases, and to reliably predict the presence and extent of any thrombus of the vena cava. ${ }^{24}$ RCC can appear iso-, hyper-, or hypodense on uncontrasted CT scans, and usually demonstrates a significant contrast enhancement of about $115 \mathrm{HU}$ and intratumoural areas of necrosis. The detection of visceral metastases appears to be crucial since it has been shown that even patients with metastatic disease might benefit from radical nephrectomy followed by immunotherapy. Involvement of the renal vein and inferior vena cava with tumour thrombus will change surgical strategy. ${ }^{24}$ This is important in all known cases of RCC. Although comprehensive renal mass evaluation protocol provides a more thorough patient evaluation, only a small fraction of indeterminate renal masses seen on US are malignant and a targeted renal CT imaging protocol is suggested for evaluation of indeterminate renal masses incidentally discovered on US. ${ }^{25}$

In one of our patients hydronephrosis was present but a possible level of obstruction not identified. The radiologist however would notice the hydonephrosis and suggest lower cuts to identify the collecting system (especially in view of an US indicating hydronephrosis). In standard renal imaging, the pelvis is only included in cases of possible renal calculi and staging of known renal masses (not suspected renal lesions). ${ }^{4,26-29}$

\section{Conclusion}

In this study only 3 of 133 cases demonstrated significant pelvic abnormalities on CT scans done for specific upper-abdominal pathol- ogy. Masking of the pelvis did not influence the outcome of the final diagnosis, and therefore it can be concluded that it is not essential to include the pelvis in the field of radiation in these cases.

The literature however recommends the inclusion of the pelvis in the following scenarios:

1. Staging of a known renal mass. The collecting system must be imaged in the event of a possible transitional carcinoma.

2. Imaging of renal calculi.

3. Some authors include the pelvis with assessment of liver metastases. The value of US investigation as a sifting tool may be underestimated and its value needs to be evaluated formally.

1. Cohnen M, Poll LJ, Puettmann C, Ewen K, Saleh A, Modder U. Effective doses in standard protocols for multi-slice CT scanning. Eur Radiol 2003; 13: 1148-1153.

2. Oh KY, Gilfeather M, Kennedy A, et al. Limited abdominal MRI in the evaluation of acute right upper quadrant pain. Abdom Imaging 2003; 28: 643-651.

3. Ramsay DW, Markham DH, Morgan B, Rodgers PM, Liddicoat AJ. The use of dilute Calogen as a fat density oral contrast medium in upper abdominal computed tomography, compared with the use of water and positive oral contrast media. Clin Radiol 2001; 56: 670-673.

4. Tomiak MM, Foley WD, Jacobson DR. Variable-mode helical CT: imaging protocols. Am J Roentgenol 1995; 164: 1525-1531.

5. O'Malley ME, Halpern E, Mueller PR, Gazelle GS. Helical CT protocols for the abdomen and pelvis: a survey. Am J Roentgenol 2000; 175: 109-113.

6. Federle MP, Blachar A. CT evaluation of the liver: principles and techniques. Semin Liver Dis 2001; 21: $135-145$.

7. Benson M, Bree RL, Schwab RE, Ouimette M. Computed tomographic studies of the painful abdomen. Radiology 1985; 155:443-444.

8. Foley WD, Mallisee TA, Hohenwalter MD, Wilson CR, Quiroz FA, Taylor AJ. Multiphase hepatic CT with a multirow detector CT scanner. Am J Roentgenol 2000; 175:679-685.

9. Ward M. Upper abdominal pain-management issues. Aust Fam Physician 1994; 23:331-336.

10. Frush DP. Review of radiation issues for computed tomography. Semin Ultrasound CT MR 2004; 25(1): 17-24.

11. Niu L, Hao Y, Zhou C, Dai J. Diagnostic significance of ultrasonography and CT for large upper abdominal mass. Chin Med J (Engl) 2002; 115: 1358-1362.

12. Ditchfield MR, De Campo JF, Waters KD, Nolan TM. Wilms' tumour: a rational use of preoperative imaging. Med Pediatr Oncol 1995; 24:93-96.

13. Poletti PA, Kinkel K, Vermeulen B, Irmay F, Unger PF, Terrier F. Blunt abdominal trauma: should US be used to detect both free fluid and organ injuries? Radiology 2003; 227(1): 95-103.

14. Lentz KA, McKenney MG, Nunez DB JR, Martin L. Evaluating blunt abdominal trauma: role for ultrasonography. J Ultrasound Med 1996; 15: 447-451.

15. Foley WD, Kerimoglu Ulku. Abdominal MDCT: Liver, pancreas and biliary tract. Semin Ultrasound CT MR 2004; 25: 122-144.

16. Schima W, Ba-Ssalamah A, Kolblinger C, Kulinna-Cosentini C, Puespoek A, Gotzinger P. Pancreatic adenocarcinoma. Eur Radiol 2006, epub ahead of publication.

17. Gritzmann N, Macheiner P, Hollerweger A, Hubner E. CT in the differentiation of pancreatic neoplasms - progress report. Dig Dis 2004; 22(1): 6-17.

18. Slattery JM, Sahani DV. What is the current state-of-the-art imaging for detection and staging of cholangiocarcinoma? Oncologist 2006; 11: 913-922.

19. Soyer P, Bluemke DA, Reichle R,et al. Imaging of intrahepatic cholangiocarcinoma: 1.Peripheral cholangiocarcinoma. Am J Roentgenol 1995; 165: 1427-1431

20. Soyer P, Bluemke DA, Reichle R, et al. Imaging of intrahepatic cholangiocarcinoma: 2.Hilar cholangiocarcinoma. Am J Roentgenol 1995; 165: 1433-1436.

21. Byun JH. Radiological staging of hilar cholangiocarcinoma. Korean J Gastroenterol 2005; 46(1): 7-15.

22. Spiral CT protocols. http//www.ctisus.org.

23. Kim J. Imaging findings of renal cell carcinoma. Expert Rev Anticancer Ther 2006; 6: 895-904.

24. Heidenreich A, Ravery V, European Society of Oncological Urology. World J Urol 2004; 22:307-315.

25. Prasad SR, Saini S, Stewart S, Hahn PF, Halpern EF. CT characterization of 'indeterminate' renal masses: targeted or comprehensive scanning? J Comput Assist Tomogr 2002; 26:725-727.

26. Bosniak MA. The small $(<3.0 \mathrm{~cm})$ renal parenchymal tumour; detection, diagnosis, and controversies. Radiology 1991; 179:307-317.

27. Davidson AJ, Hartman DS, Choyke PL, Wagner B. Radiologic assessment of renal masses; implications for patient care. Radiology 1997; 202:297-305.

28. McNicholas MM, Raptopoulos VD, Schwartz RK, et al. Excretory phase CT urography for opacification of the urinary collecting system. Am J Roentgenol 1998; 170:1261-1267.

29. Kassouf W, Aprikian AG, Laplante M, Tanguay S. Natural history of renal masses followed expectantly. J Urol 2004; 171(1): 111-113. 